\title{
Effect of a short-term exercise program on glycemic control measured by fructosamine test in type 2 diabetes patients
}

\author{
Bruno P Moura', Paulo RS Amorim", Bruno PP Silva', Sylvia CC Franceschini', Janice S Reis ${ }^{3}$ and João CB Marins ${ }^{1}$
}

\begin{abstract}
Background: Glycated hemoglobin (A1C) and Fasting Plasma Glucose (FPG) are the two monitoring blood glucose tests most frequently used. However, both methods are shown to be insensitive to detect glycemic variations in short duration periods. Therefore, we aimed to assess the effect of a short-term exercise program on glycemic levels measured by fructosamine concentrations in type 2 diabetes patients.

Methods: Eight volunteers (51.1 \pm 8.2 years) underwent a supervised exercise program during eight weeks (3 d.wk ${ }^{-1}, 50-60 \%$ of $\mathrm{VO}_{2}$ peak for 30-60 minutes). The body composition, $\mathrm{VO}_{2}$ peak, A1C, FPG, fructosamine and capillary blood glucose (CBG) were evaluated. We used ANOVA - One Way for repeated measures followed by Tukey post-hoc test and paired t test. $P$ values $<0.05$ were considered significant.

Results: We found statistical differences on the concentrations of fructosamine, $\mathrm{VO}_{2}$ peak and CBG. However, $\mathrm{A} 1 \mathrm{C}$ and FPG showed no statistical difference. Fructosamine declined by 15\% (57 $\mu \mathrm{mol} / \mathrm{L})$ between the beginning and the end of the study. Individually, $50 \%$ of the sample reached the reference values for the normality in fructosamine test. $\mathrm{VO}_{2}$ peak increased by $14.8 \%\left(3.8 \mathrm{ml} \cdot \mathrm{kg}^{-1} \cdot \mathrm{min}^{-1}\right)$ and $\mathrm{CBG}$ decreased on an average of $34.4 \%(69.3 \mathrm{mg} / \mathrm{dL})$.

Conclusions: Fructosamine test is effective in the evaluation of glucose with type 2 diabetes patients when undergoing a short exercise program, alternatively to the traditional A1C and FPG assessment. Our results are relevant in clinical practice, because the significant improvement in glycemic status can help to evaluate the inclusion of exercise as adjunct therapy to replace the prescription of additional drugs in poorly controlled patients.
\end{abstract}

Keywords: Diabetes management, Physical exercise, Glucose assessment, Aerobic exercise, Cycle ergometers, Cardiorespiratory fitness

\section{Background}

Glycemic control is a major therapeutic goal for type 2 diabetes patients. The deleterious effects of glucotoxicity have an important role in the progressive impairment of insulin secretion and sensitivity, two major factors in the pathogenesis of type 2 diabetes, which can lead to future microvascular diseases or others complications [1]. Studies have shown that glycemic control is the key to diabetes control, because it is associated with significant reductions of microvascular complication rates (retinopathy and nephropathy) and neuropathy [2].

\footnotetext{
* Correspondence: pramorim@ufv.br

'Department of Physical Education, Human Performance Laboratory,

Universidade Federal de Viçosa, Viçosa, Minas Gerais, Brazil

Full list of author information is available at the end of the article
}

One way to achieve a good glycemic control is through exercise [3] that has a well-known effect on type 2 diabetes [4]. Thus exercise has been recommended for diabetics because increase glucose uptake to active muscle and decrease the risk of cardiovascular diseases $[5,6]$.

Glycated hemoglobin (A1C) and Fasting Plasma Glucose (FPG) are the most common analysis for monitoring blood glucose levels [2]. However, A1C reflects a glycemic control over a period of $2-3$ months, which is too long to assess the effectiveness of short-term exercise programs on glycemic control [7]. Likewise, the FPG is very variable because is influenced by the glycemic content ingested in the diet from the previous night [8].

Fructosamine (measure of glycated proteins, specifically albumin) has a half-life shorter than the red cell. Therefore 
the fructosamine is presented as an indicative of glycemic control on period of 2-3 weeks [9]. Thus, this test is particularly useful when assessing periods of rapid change in glycemia [10], such as those resulting from a short-term exercise programs $[9,11,12]$.

Therefore, we aimed to assess the effect of a short-term exercise program on glycemic levels measured by fructosamine concentrations in type 2 diabetes patients.

\section{Methods}

\section{Patients}

Patients recruitment for this study occurred through advertisements in local media, which were selected according to the following inclusion criteria: a) Have type 2 diabetes diagnosed by criteria of the American Diabetes Association (ADA) [2]; b) Must not have diabetes complications (cardiovascular disease, neuropathy, retinopathy and nephropathy); c) Must not have practiced physical exercises with professional guidance in the last 2 months preceding the start of the program; d) Agreed not to do other kinds of physical exercises with professional guidance in addition to those on the program during the study and maintain their usual diets throughout the intervention period.

Twenty volunteers signed up to participate in the study, fifteen of which were approved by inclusion criteria. After a meeting to explain all the study procedures, eleven patients confirmed their participation. After the first week of the study, one was excluded due to an impediment in performing physical exercise because of ulceration on the feet. Two other gave up during the exercise program for personal reasons. Finally, eight patients (average age 51.1 \pm 8.2 years, diagnosis: $5.1 \pm 4.6$ years ago), four of whom were women, composed the final sample. All patients were on use of medications since diagnosis of diabetes (metformin, insulin, and glimepiride). No patient reported changing in his dose of medication during the study. Though, recent study showed that metformin did not significantly attenuate the benefits of exercise on glycemic control or fitness [13].

All patients enrolled in this study had the approval of their physician to practice physical exercise of moderate intensity and signed terms of consents. The study was approved by the Ethics Committee on Human Research of Universidade Federal de Viçosa.

\section{Anthropometry and body composition assessment}

Anthropometric measurements were obtained at the Human Performance Laboratory (LAPEH), by a trained examiner. Weight, height and waist circumference measurements were performed by calibrated equipment based on the procedures described by Lohman et al. [14]. Body mass index (BMI) was calculated and analyzed according to guidelines of the World Health Organization (WHO) [15]. Body composition assessment was performed by the apparatus Body Composition Analyser (BIA 310 bioimpedance analyzer, Biodynamics Corp.) [16].

\section{Biochemical tests}

Blood sample collection was obtained from the median cubital vein after eight hours fasting by a trained biochemist. Technique of vacuum collection tubes with EDTA-K $\mathrm{K}_{3}$ was used for hematology (A1C and Fasting Plasma Glucose) and tubes with clot accelerator $\left(\mathrm{SiO}_{2}\right)$ and gel separator was used for serology (Fructosamine). The samples were analyzed in the Clinical Laboratory, Division of Health, at Universidade Federal de Viçosa. The following methods were used for analysis: a) Glycated Haemoglobin (A1C): HPLC (High Performance Liquid Chromatography), by the device VARIANT II System (Bio-Rad Laboratories, Inc., USA) with reference value (RV) for normality $\leq 6.5 \%$ [2]; b) Fructosamine: colorimetric with reduction of nitroblue tetrazolium (NBT) by device Modular (Roche), with RV for normal 205-285 $\mu \mathrm{mol} / \mathrm{L}$ and c) Fasting Plasma Glucose (FPG): glucose oxidase method in device Cobas Mira Plus (Roche), with RV for diabetes $\geq 126 \mathrm{mg} / \mathrm{dL}(7.0 \mu \mathrm{mol} / \mathrm{L})$ [2]. Capillary Blood Glucose (CBG) was measured, before and after each exercise session, by blood glucose monitor Accu-Chek Go (Roche).

\section{Cardiorespiratory fitness}

Cardiorespiratory fitness of patients was measured by the metabolic gas analyzer VO2000 (Medical Graphics Corporation) and analyzed by software Aerograph 4.3 (Medical Graphics Corporation). Metabolic gas analyzer was automatic calibrated before each test. Cardiorespiratory fitness tests were performed on a cycle ergometer (ISO1000, SCIFIT $^{\bullet}$ Corporate Headquarters). All necessary precautions for to perform cardiorespiratory fitness tests in diabetic patients (e.g. blood pressure measurement at rest and during testing, measurement of blood glucose before and after the test) was taken. Prior to cardiorespiratory fitness tests, patients performed an adaptation to cycle ergometer through a warm-up five minutes. Ramp protocol was used with increments to charge by the minute; in which patients were encouraged to reach $85 \%$ of heart rate (HR) estimated by $\mathrm{HR}_{\text {max. }}=208-($ age $\times 0.7)$ proposed by Tanaka et al. [17]. Because we do not have the presence of a physician during the test of cardiorespiratory fitness, for security reasons, when $85 \%$ of maximal HR was reached, the cardiorespiratory fitness test was interrupted and started the recovery stage. Test data ( $\mathrm{HR}$ and $\mathrm{VO}_{2}$ ) were used as a basis for the development of individual equations to estimate peak oxygen consumption $\left(\mathrm{VO}_{2}\right.$ peak) by linear regression (SigmaPlot ${ }^{\circ}$ Version 11.0; Systat Software, Inc., Chicago, IL, USA) [18].

\section{Protocol}

The study lasted ten weeks, in which eight weeks were intervention with physical exercise (Table 1). The exercises 
Table 1 Drawing of the progression of the study

\begin{tabular}{|c|c|c|c|c|c|c|c|c|c|c|}
\hline Weeks & $1^{\mathrm{Ev}}$ & 2 & 3 & 4 & $5^{\text {Fru }}$ & 6 & 7 & 8 & 9 & $10^{\mathrm{Ev}}$ \\
\hline Exercise period & \multicolumn{10}{|c|}{ Exercise } \\
\hline Intensities & & 50 & 50 & 55 & 60 & 60 & 60 & 60 & 60 & \\
\hline Duration/session & & 30 & 40 & 50 & 60 & 60 & 60 & 60 & 60 & \\
\hline
\end{tabular}

Legend: Ev = Evaluation (Anthropometry and body composition, biochemical and cardiorespiratory fitness tests); Fru = Fructosamie examination during exercise intervention); Intensities = percentage of $\mathrm{VO}_{2}$ peak and Duration/ Session $=$ Minutes

were performed into Human Performance Laboratory, at Universidade Federal de Viçosa, by cycle ergometers (Cycle 167, 2001, ERGO-FIT ${ }^{\circ}$ ). Physical exercises were performed three days per week (d.wk ${ }^{-1}$ ) over eight weeks. Throughout the study, duration bout ranged from 30 to 60 minutes and intensity ranges from $50 \%$ to $60 \%$ of $\mathrm{VO}_{2}$ peak (Table 1 ). Training bout was divided into three phases: warm-up, physical exercise and cool down. The warm-up and cool down always lasted five minutes each. Intensity control based on $\mathrm{VO}_{2}$ peak was performed by monitoring the corresponding HR, through the HR monitor (Polar RS800CX, Finland). Patients were asked to maintain an average speed of $20 \mathrm{~km} / \mathrm{h}$ throughout the exercise and the load (watts) on the cycle ergometer was increased up to achieve the target $\mathrm{HR}$ based on the percentage of $\mathrm{VO}_{2}$ peak for the specific bout. However, if a participant presented peripheral fatigue, the load (watts) on the cycle ergometer was reduced and the exercise speed has increased, in order to maintain physiological load (HR) required. The total load of the session (Figure 1) was calculated by the following equation: Total load of session $=$ Intensity $\times$ Duration [19]. Fructosamine analysis was performed pre, during and post study (Weeks 1, 5 and 10) (Table 1). We do not control food intake of the patients and they were asked to maintain their usual diet throughout the physical exercise period.

\section{Statistics}

All variables were approved by normality test (Shapiro-Wilk) and Equal Variance test. Thus, results are presented as mean $(\bar{x})$ and standard deviation (SD). ANOVA - One Way for repeated measures followed by Tukey post-hoc test was used to assess the evolution of fructosamine during the study. For the other variables, paired $t$ test was used to compare the performance pre and post intervention, and pre and post exercise session for the capillary blood glucose. $P$ values $<0.05$ were considered significant. The tests were performed by the software SigmaPlot Version 11.0 (Systat Software, Inc., Chicago, IL, USA).

\section{Results}

We found significant statistical differences on fructosamine concentrations between the study periods $(\mathrm{P}$ values $=0.05$; Power of performed test $=0.85-$ Figure 2 ), $\mathrm{VO}_{2}$ peak (Table 2) and CBG (Figure 1) before and after exercise sessions.

Fructosamine test showed a decrease in blood concentration of $15 \%(57 \mu \mathrm{mol} / \mathrm{L})$ between the beginning and the end of the study, while $\mathrm{VO}_{2}$ peak showed an increase of $14.8 \%$ $\left(3.8 \mathrm{ml} \cdot \mathrm{kg}^{-1} \cdot \mathrm{min}^{-1}\right)$. Capillary blood glucose during exercise sessions showed an average decrease of $34.4 \%(69.3 \mathrm{mg} / \mathrm{dL})$.

Anthropometric variables, body composition, A1C and FPG showed no statistical difference between pre and post study periods (Table 2). However, when assessing the BMI, according to World Health Organization (WHO) [15], the classification of the subjects were in the state of obesity class I, confirmed by the high percentage of body fat measured by bio impedance technique.

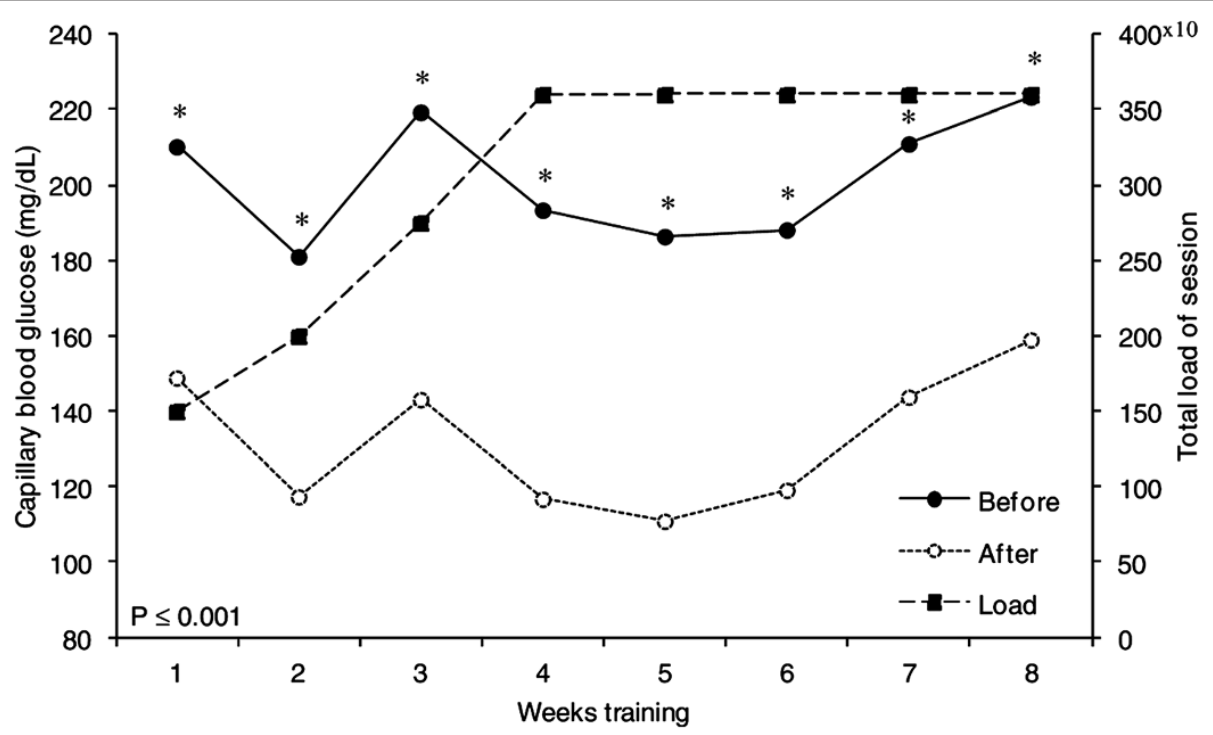

Figure 1 Average weekly blood glucose before and after exercise sessions and the evolution of training load. 


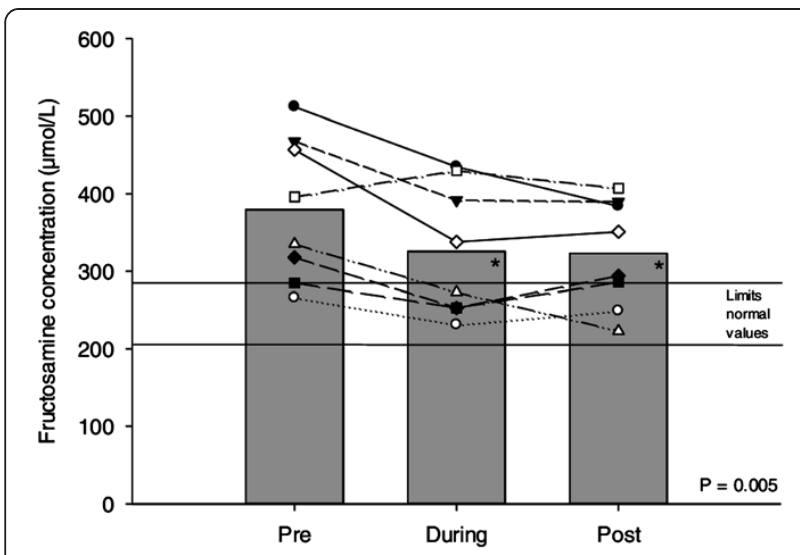

Figure 2 Mean concentration of fructosamine pre, during and post study.

\section{Discussion}

Evidences from this study support the use of fructosamine test to assess the effect of a short-term exercise program on glycemic control in type 2 diabetes patients. This test showed a decrease of $14.2 \%$ from the first to fifth week of the study (after three exercise weeks) and $0.8 \%$ from the fifth to tenth week (Figure 2).

Individually, $50 \%$ of the sample reached RV. Two subjects reached the RV in the fifth week and remained in that range until the end of the study, while two others reached the RV in the fifth week but failed to keep them until the end of the study. These results are relevant in clinical practice, because the significant improvement of $15 \%$ in glycemic status after eight weeks of physical exercise can help to evaluate the exercise inclusion as adjunct therapy to replace the prescription of additional drugs in poorly controlled patients.

Studies has recommended the use of fructosamine test for glycemic control in short periods of monitoring, which may occur fast changes of blood glucose levels [7,9,20-24].

Table 2 Anthropometric variables, body composition, A1C, FPG and $\mathrm{VO}_{2}$ peak of subjects pre $(n=8)$ and post ( $n=8)$ study

\begin{tabular}{|c|c|c|c|}
\hline Variables & $\begin{array}{c}\text { Pre } \\
\bar{x} \pm \text { SD }\end{array}$ & $\begin{array}{c}\text { Post } \\
\bar{x} \pm \text { SD }\end{array}$ & $P$ values \\
\hline Weight (kg) & $83.7 \pm 17.1$ & $83.2 \pm 17.7$ & 0.195 \\
\hline BMI $\left(\mathrm{kg} / \mathrm{m}^{2}\right)$ & $30.2 \pm 6.4$ & $29.8 \pm 6.2$ & 0.063 \\
\hline Waist circumference $(\mathrm{cm})$ & $102.6 \pm 12.0$ & $97.5 \pm 20.1$ & 0.312 \\
\hline$\%$ Fat & $33.4 \pm 6.3$ & $32.4 \pm 5.9$ & 0.245 \\
\hline Fat mass $(\mathbf{k g})$ & $28.6 \pm 9.8$ & $27.4 \pm 9.3$ & 0.137 \\
\hline $\mathrm{A} 1 \mathrm{C}(\%)$ & $8.9 \pm 1.6$ & $8.3 \pm 1.8$ & 0.083 \\
\hline FPG (mg/dL) & $192.2 \pm 73.9$ & $176.7 \pm 55.1$ & 0.459 \\
\hline Peak VO $\mathrm{V}_{2}\left(\mathrm{ml} . \mathrm{kg} \cdot \mathrm{min}^{-1}\right)$ & $25.7 \pm 5.1$ & $29.5 \pm 6.6$ & 0.048 \\
\hline
\end{tabular}

Legend: $\mathrm{BMI}=$ Body Mass Index; \% Fat. = Body Fat Percentage; A1C = Glycated hemoglobin e FPG = Fasting Plasma Glucose. Data are showed as mean $(\bar{x}) \pm$ Standard Deviation (SD).
Raz et al. [12] investigated the influence of 12 weeks moderate exercise on the parameters of glycemic control in type 2 diabetes patients and found a significant reduction in the fructosamine levels. Unlike the studies cited above, the current manuscript assessed the effect of short-term exercise program on glycemic control measured by fructosamine test. Taken together, results from these studies provide evidence for the use of fructosamine test for glycemic control short-term exercise program.

Rychlewski e Szczesniak [11] investigated the effects of regular exercise along three weeks on fructosamine levels in children with type 1 diabetes. They found a reduction in the frutosamine levels, as well as increased insulin sensitivity. Although the results presented above involve patients with type 1 diabetes, they are quite similar to the ones presented in the current study, involving type 2 diabetes patients. However, the improvement of glycemic control observed could likely be maximized with the continuation of the exercise program in conjunction with new increments in the training loads.

The importance of fructosamine test as the method of measuring glycemic status during short-term exercise program is most apparent when viewed in conjunction with the evaluation of A1C and FPG (Table 2). Both tests often used as a way of controlling the glycemic levels, despite showing a reduction that can be considered clinically important, showed no statistically significant differences before and after study. The traditional tests (A1C and FPG) used in the assessment of glycemic control showed a lower sensitivity to indicate the beneficial changes induced by exercise, as assessed by fructosamine test. This fact may explain unsatisfactory results of the effect of a short-term exercise program on glycemic control sometimes found in the literature $[25,26]$.

In a study conducted in a developing country, the use of fructosamine test presented itself as a good alternative for the A1C test [27]. This test may be particularly appropriate for developing countries like Brazil, where the supply of strips for blood glucose control is not accessible to type 2 diabetes patients that are not being insulinized.

After the fourth exercise week, the training load remained unaffected (Figure 1). Because of a difficulty in generating incremental load due to the fact that diabetic patients has a reduced fitness [5]. However, even with the maintenance of training load, the reduction in CBG after weekly exercise sessions remained statistically significant $(\mathrm{P} \leq 0.001)$ until the end of the exercise program.

Evolution of training load in relation to fructosamine test results, it appears that fructosamine concentration (Figure 2) remained practically unchanged after the stabilization of the workload. This fact can be explained by the physiological adaptations triggered by the exercise in the body and shows need of workload increases to ensure the continuity of the beneficial effects of exercise 
on glycemic control. However, even with the difficulty of increasing training loads, after the fourth exercise week, the subjects showed an improvement in cardiorespiratory fitness (Table 2). These results confirm that the intensity used in the exercise program was adequate to cause physiological adaptations.

The lack of alteration of anthropometric variables and body composition presented in the current study are corroborated by the study of Boulé et al. [28]. These authors conducted a meta-analysis about exercise effects on glycemic control and body mass in type 2 diabetes patients. They found that exercise effects on glycemic control are independent of weight loss.

The main limitations of this study were the small sample size and the absence of a control group. These facts occurred due to the difficulty in finding volunteers with type 2 diabetes without microvascular diseases or others complication, able and willing to participate in a program of controlled exercise for eight weeks. However, all variables were approved by the normality test and present parametric distribution. Another limitation was the lack of control over food intake, despite recommendations to maintain their usual diets are continually enforced on all subjects throughout the intervention period.

Thus, addition to sensitivity in detecting the effects of a short-term exercise program on glycemic control, fructosamine test has additional advantages. Such as realization in any day time without the need to fasting, low cost, as well as the fact that there are available domestic monitoring devices with the validity and accuracy evidenced in relation to laboratory tests [29]. A key aspect to type 2 diabetes patients that should be highlighted is the existence of studies linking the reduced fructosamine levels with decreased cardiovascular risk and mortality rates $[10,24]$.

\section{Conclusions}

The results of our study support the use of fructosamine test to assess the effect of a short-term exercise program on glycemic control in type 2 diabetes patients. This test showed as a good alternative compared to traditional measures of glycemic control (A1C and FPG).

Our results are relevant in clinical practice, because the significant improvement in glycemic status after eight weeks of physical exercise can help to evaluate the inclusion of exercise as adjunct therapy to replace the prescription of additional drugs in poorly controlled patients.

Nevertheless, further studies with a larger sample, diet control and exercise programs with constant workloads increments should be performed to replicate the results verified in our study.

\section{Competing interests}

The authors declare that they have no competing interests.

\section{Authors' contributions}

BPM and PRSA were responsible for the study design. BPPS was responsible for overseeing the exercise training of volunteers. JCBM, SCCF and JSR were co-advisors and made corrections in the text. All authors read and approved the final paper.

\section{Acknowledgements}

We thank FAPEMIG for the scholarship to BPM, CNPq for scholarship to BPPS and the FUNARPÓS for financing the procedures performed in this study. In addition, we thank the volunteers who kindly participated in the study.

\section{Funding}

FAPEMIG (Foundation for Research Support of Minas Gerais State); CNPq (The National Council for Scientific and Technological Development) and FUNARPÓS (FUNARBE Program of Support the Graduate of Universidade Federal de Viçosa).

\section{Author details}

'Department of Physical Education, Human Performance Laboratory, Universidade Federal de Viçosa, Viçosa, Minas Gerais, Brazil. ${ }^{2}$ Department of Nutrition, Universidade Federal de Viçosa, Viçosa, Minas Gerais, Brazil. ${ }^{3}$ Institute of Education and Research of Santa Casa de Belo Horizonte, IER-SCBH, Belo Horizonte, Minas Gerais, Brazil.

Received: 15 October 2013 Accepted: 22 January 2014

Published: 11 February 2014

\section{References}

1. Giaccari A, Sorice G, Muscogiuri G: Glucose toxicity: the leading actor in the pathogenesis and clinical history of type 2 diabetes - mechanisms and potentials for treatment. Nutr Metab Cardiovasc Dis 2009, 19:365-377.

2. American Diabetes Association: Standards of medical care in diabetes-2011. Diabetes Care 2011, 34(Suppl 1):S11-S61.

3. Moura BP, Natali AJ, Marins JCB, Amorim PRS: Different approaches of physical training used in the management of type 2 diabetes: a brief systematic review of randomised clinical trials. Brit J Diabetes Vasc Dis 2011, 11:210-216.

4. Colberg SR, Sigal RJ, Fernhall B, Regensteiner JG, Blissmer BJ, Rubin RR, Chasan-Taber L, Albright AL, Braun B: Exercise and type 2 diabetes: the American College of Sports Medicine and the American Diabetes Association: joint position statement. Diabetes Care 2010, 33:e147-e167.

5. McGavock JM, Eves ND, Mandic S, Glenn NM, Quinney HA, Haykowsky MJ: The role of exercise in the treatment of cardiovascular disease associated with type 2 diabetes mellitus. Sports Med 2004, 34:27-48.

6. Marwick TH, Hordern MD, Miller T, Chyun DA, Bertoni AG, Blumenthal RS, Philippides G, Rocchini A: Exercise training for type 2 diabetes mellitus: impact on cardiovascular risk: a scientific statement from the American Heart Association. Circulation 2009, 119:3244-3262.

7. Hom F: Fructosamine, hemoglobin A1C, and measures of diabetic control. Diabetes Technol Ther 1999, 1:443-445.

8. Liu K, Stamler J, Stamler R, Cooper R, Shekelle RB, Schoenberger JA, Berkson DM, Lindberg HA, Marquardt J, Stevens E, Tokich T: Methodological problems in characterizing an individual's plasma glucose level. J Chronic Dis 1982, 35:475-485.

9. Klonoff DC: Serum fructosamine as a screening test for type 2 diabetes. Diabetes Technol Ther 2000, 2:537-539.

10. Misciagna $G$, De Michele G, Trevisan M: Non enzymatic glycated proteins in the blood and cardiovascular disease. Curr Pharm Des 2007, 13:3688-3695.

11. Rychlewski T, Szczesniak L: [Fructosamine in blood serum, binding and degradation of $125 \mathrm{~J}$-insulin by erythrocyte receptors in young persons with type I diabetes-effect of physical exercise]. Pol Arch Med Wewn 1996, 95:212-217

12. Raz I, Hauser E, Bursztyn M: Moderate exercise improves glucose metabolism in uncontrolled elderly patients with non-insulin-dependent diabetes mellitus. Isr J Med Sci 1994, 30:766-770.

13. Boule NG, Kenny GP, Larose J, Khandwala F, Kuzik N, Sigal RJ: Does metformin modify the effect on glycaemic control of aerobic exercise, resistance exercise or both? Diabetologia 2013, 56(11):2378-2382.

14. Lohman TG, Roche AF, Martorell R: Anthropometric standardization reference manual. Champaign, IL: Human Kinects; 1988. 
15. WHO: World Health Organization. Diet, nutrition and the prevention of chronic diseases. World Health Organ Tech Rep Ser 2003, 916:1-149.

16. Lukaski HC, Bolonchuk WW, Hall CB, Siders WA: Validation of tetrapolar bioelectrical impedance method to assess human body composition J Appl Physiol 1986, 60:1327-1332.

17. Tanaka H, Monahan KD, Seals DR: Age-predicted maximal heart rate revisited. J Am Coll Cardiol 2001, 37:153-156.

18. Ekelund U, Franks PW, Sharp S, Brage S, Wareham NJ: Increase in physical activity energy expenditure is associated with reduced metabolic risk independent of change in fatness and fitness. Diabetes Care 2007, 30:2101-2106.

19. Caballero JAR, Manso JMG, Valdivielso MN, Caballero JAR: Bases teoricas del entrenamiento deportivo. 1st edition. Madrid: S.L. Editorial Gymnos; 1996.

20. Post EM, Moore JD, Ihrke J, Aisenberg J: Fructosamine levels demonstrate improved glycemic control for some children attending a diabetes summer camp. Pediatr Diabetes 2000, 1:204-208.

21. Gomo Z: Serum fructosamine: a parameter for monitoring metabolic control in diabetes. Cent Afr J Med 1992, 38:358-362.

22. Yahaya IA, Isah HS, Anaja PO: Serum fructosamine in the assessment of glycaemic status in patients with sickle cell anaemia. Niger Postgrad Med J 2006, 13:95-98.

23. Lindsey CC, Carter AW, Mangum S, Greene D, Richardson A, Brown SJ, Essary JL, McCandless B: A prospective, randomized, multicentered controlled trial to compare the annual glycemic and quality outcomes of patients with diabetes mellitus monitored with weekly fructosamine testing versus usual care. Diabetes Technol Ther 2004, 6:370-377.

24. Browner WS, Pressman AR, Lui LY, Cummings SR: Association between serum fructosamine and mortality in elderly women: the study of osteoporotic fractures. Am J Epidemiol 1999, 149:471-475.

25. Snowling NJ, Hopkins WG: Effects of different modes of exercise training on glucose control and risk factors for complications in type 2 diabetic patients: a meta-analysis. Diabetes Care 2006, 29:2518-2527.

26. Bello Al, Owusu-Boakye E, Adegoke BO, Adjei DN: Effects of aerobic exercise on selected physiological parameters and quality of life in patients with type 2 diabetes mellitus. Int I Gen Med 2011, 4:723-727.

27. Islam N, Akhter J, Kayani N, Khan MA: Fructosamine: an alternative assessment of past glycaemic control in developing countries. J Pak Med Assoc 1993, 43:238-240.

28. Boule NG, Haddad E, Kenny GP, Wells GA, Sigal RJ: Effects of exercise on glycemic control and body mass in type 2 diabetes mellitus: a meta-analysis of controlled clinical trials. JAMA 2001, 286:1218-1227.

29. Edelman SV, Callahan P, Deeb LC: Multisite evaluation of a new diabetes self-test for glucose and glycated protein (fructosamine). Diabetes Technol Ther 2000, 2:233-238.

doi:10.1186/1758-5996-6-16

Cite this article as: Moura et al: Effect of a short-term exercise program on glycemic control measured by fructosamine test in type 2 diabetes patients. Diabetology \& Metabolic Syndrome 2014 6:16.

\section{Submit your next manuscript to BioMed Central and take full advantage of:}

- Convenient online submission

- Thorough peer review

- No space constraints or color figure charges

- Immediate publication on acceptance

- Inclusion in PubMed, CAS, Scopus and Google Scholar

- Research which is freely available for redistribution 